Check for updates

Cite this: RSC Adv., 2018, 8, 4842

\title{
Systematic investigation of functional ligands for colloidal stable upconversion nanoparticles $\uparrow$
}

\author{
Hien T. T. Duong, (D) $\ddagger^{\star a}$ Yinghui Chen, ${ }^{\text {ab }}$ Sherif Abdulkader Tawfik, (D) Shihui Wen, (D) a \\ Maryam Parviz, ${ }^{\mathrm{ab}}$ Olga Shimoni ${ }^{\mathrm{ab}}$ and Dayong Jin (D) ab
}

Despite intense efforts on surface functionalization to generate hydrophilic upconversion nanoparticles (UCNPs), long-term colloidal stability in physiological buffers remains a major concern. Here we quantitatively investigate the competitive adsorption of phosphate, carboxylic acid and sulphonic acid onto the surface of UCNPs and study their binding strength to identify the best conjugation strategy. To achieve this, we designed and synthesized three di-block copolymers composed of poly(ethylene glycol) methyl ether acrylate and a polymer block bearing phosphate, carboxylic or sulphonic acid anchoring groups prepared by an advanced polymerization technique, Reversible Addition Fragmentation Chain Transfer (RAFT). Analytical tools provide the evidence that phosphate ligands completely replaced all the oleic acid capping molecules on the surface of the UCNPs compared with incomplete ligand exchange by carboxylic and sulphonic acid groups. Meanwhile, simulated quantitative adsorption energy measurements confirmed that among the three functional groups, the calculated adsorption strength for phosphate anchoring ligands is higher which is in good agreement with experimental results regarding the best colloidal stability, especially in phosphate buffer solution. This finding suggests that polymers with multiple anchoring negatively charged phosphate moieties provide excellent colloidal stability for lanthanide ion-doped luminescent nanoparticles for various potential applications.

Received 30th December 2017 Accepted 17th January 2018

DOI: $10.1039 / c 7 r a 13765 f$

rsc.li/rsc-advances other luminescent materials (e.g. organic dyes, quantum dots and luminescent metal nanoclusters). ${ }^{10-12}$ In addition, the use of NIR light allows the deeper penetration in biological tissues that is highly favourable for many biomedical applications. NIR light penetrates tissue approximately $23 \mathrm{~cm}$ in depth. ${ }^{13}$ Over the years, UCNPs with precisely controlled size, shape and compositions have been prepared in high boiling point organic solvent. ${ }^{14}$ Nonetheless, for the UCNPs to become practically useful in bioapplications, it requires to overcome a critical challenge of surface functionalisation that will transform UCNPs to be stable in physiological environment and specific to recognize the target biomolecules.

The as-synthesized UCNPs are hydrophobic in nature due to their capping by long-chain hydrophobic molecules (e.g. oleic acid). Typically, to yield well-dispersed nanoparticles in aqueous media for bioimaging, drug delivery and other bioanalytical applications, several methods of subsequent surface modification are applied including inorganic shell, bilayer coating, ligand oxidation and ligand exchange. ${ }^{15}$ Despite multiple studies have been done to develop a generic approach for surface functionalization, stability of UCNPs in aqueous media, especially in phosphate buffer and physiological environment still remains as a major challenge. ${ }^{16}$

Among surface modification methods, polymers containing single or multiple anchoring ligands, such as hydroxyl, amine, carboxylic acid, sulphonic acid, phosphonate and phosphate 
groups, have been extensively studied to modify the positively charged surface of UCNPs and other inorganic nanoparticles. ${ }^{15,17-19}$ However, to our best knowledge, there is no report that specifically focuses on the influence of structural variation of functional ligands on the coating density, dispersity and more importantly, the extended colloidal stability of polymer functionalized UCNPs. To fill this knowledge gap, our work provides a systematic study of competitive adsorption at the nanocrystal surface between multidentate phosphate, carboxylic and sulphonic acids with oleate ligands.

Specifically, in this paper we demonstrate that a well-defined di-block copolymer synthesized by Reversible Addition Fragmentation Chain Transfer (RAFT) technique comprising of the PEG-like polymer and plurality of functional groups as potential coordination sites for the UCNP surface. RAFT polymerization technique for polymer synthesis is very simple and versatile, provides a high control over molecular weight distribution and functional groups with no tedious synthetic process. ${ }^{20-23}$ Multiple anchoring points are demonstrated to lead to the higher coating density on the surface of the UCNPs. ${ }^{17}$ PEG-like block on the polymer serves as the outer shell on the coated UCNPs that greatly assists with colloidal stability in water, serum and aqueous buffers. PEG-like polymer also provides many advantages for biological applications, including high biocompatibility, low toxicity and immunogenicity and no metabolic degradation during clearance from the body. ${ }^{24}$ Versatility of RAFT polymerization allows introducing a second block on the copolymer containing anchoring groups for further attachment of polymer onto the surface of UCNPs. In addition, functional end-groups of the used RAFT agent and the polymers offer an opportunity for subsequent attachments of peptide, protein, antibody, folic acid, therapeutics agents and bioactive molecules. ${ }^{25-28}$

Evidently, we experimentally found and verified conclusive information on the mechanism of competitive adsorption of phosphate, carboxylic and sulphonic acids with oleate ligand by analytical methods supported by simulations. In fact, the greater adsorption energy of phosphate anchoring groups is the key mechanism for the complete replacement of hydrophilic polymers which led to the best performance of the aqueous dispersity and long-term stability of phosphate containing polymer coated UCNPs.

\section{Experimental}

\section{Materials and methods}

All chemicals were used as received from Sigma-Aldrich, unless otherwise specified. Monomer oligo(ethylene glycol) methyl ether acrylate with average $M_{\mathrm{n}}$ of $480 \mathrm{~g} \mathrm{~mol}^{-1}$ (OEGA), acrylic acid (AA), monoacryloxyethyl phosphate (MAEP), and 1-acrylamido-2-methylpropane sulphonic acid (AMPS) monomers were used as received. 2,2'-Azobisisobutyronitrile (AIBN) was purified by recrystallization twice from methanol.

\section{Synthesis of $\mathrm{NaYF}_{4}: \mathrm{Yb} / \mathrm{Er}$ upconversion nanoparticles}

The synthesis of monodisperse $\mathrm{NaYF}_{4}: \mathrm{Yb}(20 \%)$, Er (2\%) nanocrystals has been reported in the literature. ${ }^{14}$ In a typical procedure, $\mathrm{YCl}_{3}(0.78 \mathrm{mmol}), \mathrm{YbCl}_{3}(0.20 \mathrm{mmol})$, and $\mathrm{ErCl}_{3}$ $(0.02 \mathrm{mmol})$ were added to a $50 \mathrm{~mL}$ flask containing $6 \mathrm{~mL}$ of oleic acid and $15 \mathrm{~mL}$ of 1-octadecene. The mixture was heated to $160{ }^{\circ} \mathrm{C}$ for $30 \mathrm{~min}$ and then cooled to room temperature. A solution of $4 \mathrm{mmol}$ of $\mathrm{NH}_{4} \mathrm{~F}(0.1482 \mathrm{~g})$ and $2.5 \mathrm{mmol}$ of $\mathrm{NaOH}$ $(0.1 \mathrm{~g})$ in $5 \mathrm{~mL}$ of methanol was added, and then the solution was kept at room temperature for $30 \mathrm{~min}$. The mixture was then heated to $120{ }^{\circ} \mathrm{C}$ under argon for 20 min to remove methanol and water. The solution was finally heated to $300{ }^{\circ} \mathrm{C}$ under an argon atmosphere for $1.5 \mathrm{~h}$ and then cooled to room temperature. The nanocrystals were precipitated with $10 \mathrm{~mL}$ of ethanol, collected by centrifugation. The product was washed with cyclohexane, ethanol and methanol four times and the final $\mathrm{NaYF}_{4}: \mathrm{Yb} / \mathrm{Er}$ nanocrystals were re-dispersed in $10 \mathrm{~mL}$ cyclohexane for further use.

\section{Synthesis of POEGA macro-RAFT agent}

The RAFT agent, 2-(n-butyltrithiocarbonate)-propionic acid (BTPA) was prepared according to a published procedure. ${ }^{29}$ OEGA $\left(5.0 \mathrm{~g}, 1.04 \times 10^{-2} \mathrm{~mol}\right)$, RAFT agent $\left(1.13 \times 10^{-1} \mathrm{~g}\right.$, $\left.4.73 \times 10^{-4} \mathrm{~mol}\right)$ and AIBN $\left(7.77 \times 10^{-3} \mathrm{~g}, 4.73 \times 10^{-5} \mathrm{~mol}\right)$ were dissolved in $20 \mathrm{~mL}$ of toluene in a round- bottom flask equipped with a magnetic stirrer bar. The flask was then sealed with a rubber septum and purged with nitrogen gas for $30 \mathrm{~min}$. The reaction mixtures were then placed in a preheated oil bath at $70{ }^{\circ} \mathrm{C}$. After $17 \mathrm{~h}$, the polymerization was terminated by quenching the samples in an ice bath for $5 \mathrm{~min}$. The POEGA polymer was then purified three times by precipitation with excess petroleum spirits (boiling range of $40-60{ }^{\circ} \mathrm{C}$ ) followed by centrifugation (7000 rpm for $5 \mathrm{~min}$ ) and the polymer was dried under vacuum at room temperature. The samples were stored at $4{ }^{\circ} \mathrm{C}$ until required for further chain extension to form diblock copolymers. The conversion of monomer $(90 \%)$ during the course of polymerization was determined using ${ }^{1} \mathrm{H}$ NMR. The molecular weight of the POEGA macro-RAFT agent was measured to be $9800 \mathrm{~g} \mathrm{~mol}^{-1}(\mathrm{PDI}=1.10)$ by DMAc GPC and $M_{\mathrm{n}, \mathrm{NMR}}=9500 \mathrm{~g} \mathrm{~mol}^{-1}$ by ${ }^{1} \mathrm{H}$ NMR.

\section{Synthesis of POEGA- $b$-PAA, POEGA- $b$-PMAEP and POEGA- $b$ - AMPS}

POEGA with 20 repeating units $\left(M_{\mathrm{n}, \mathrm{NMR}}=9500 \mathrm{~g} \mathrm{~mol}^{-1}, M_{\mathrm{n}, \mathrm{GPC}}\right.$ $=9800 \mathrm{~g} \mathrm{~mol}^{-1}$ ) was used as a macro-RAFT agent for chain extension with three different functional monomers, acrylic acid (AA), monoacryloxyethyl phosphate (MAEP), and 2acrylamido-2-methyl-1-propanesulphonic acid (AMPS) to yield three functional polymers $\mathrm{POEGA}_{20}-b-\mathrm{PAA}_{5}, \mathrm{POEGA}_{20}-b$ -

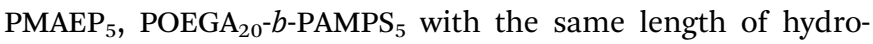
philic block (20 repeating units) and functional block (5 repeating units). The number of repeating units of POEGA was calculated from the monomer conversion obtained from ${ }^{1} \mathrm{H}$ NMR. Three reaction mixtures were prepared for the synthesis of three di-block copolymers. For the synthesis of POEGA- $b$-PAA, POEGA macro-RAFT agent $\left(1.0 \mathrm{~g}, 1.02 \times 10^{-4} \mathrm{~mol}\right)$ and AIBN $\left(3.35 \times 10^{-3} \mathrm{~g}, 2.04 \times 10^{-5} \mathrm{~mol}\right)$ were dissolved in $5 \mathrm{~mL}$ of acetonitrile and AA $\left(5.15 \times 10^{-2} \mathrm{~g}, 7.14 \times 10^{-4} \mathrm{~mol}\right)$ was added. Similar protocol was used for the synthesis of POEGA- $b$-PMAEP 
and POEGA- $b$-PAMPS with MAEP $\left(1.4 \times 10^{-1} \mathrm{~g}, 7.14 \times 10^{-4}\right.$ mol) and AMPS $\left(1.48 \times 10^{-1} \mathrm{~g}, 7.14 \times 10^{-4} \mathrm{~mol}\right)$ added. $N, N$ Dimethylformamide (DMF) was used as the solvent for the synthesis of POEGA- $b$-PAMPS due to the insolubility of AMPS monomer in acetonitrile.

The reaction mixtures were purged with nitrogen gas for $30 \mathrm{~min}$ in an ice bath. The polymerization was carried out in an oil bath at $70{ }^{\circ} \mathrm{C}$. The polymerization was terminated at the monomer conversion of around $70 \%$ by placing the samples on ice for $5 \mathrm{~min}$. The di-block copolymers were purified three times by precipitation in excess of diethyl ether followed by centrifugation (7000 rpm for $5 \mathrm{~min}$ ) and the polymer was then dried under reduced pressure at room temperature. Block copolymers with 20 repeating units of OEGA, 5 repeating units of functional monomers were obtained (as confirmed by ${ }^{1} \mathrm{H}$ NMR) was used for further comparison of grafting efficiency on the surface of upconversion nanoparticles. The molecular weight was measured to be $M_{\mathrm{n}, \mathrm{GPC}}=14700 \mathrm{~g} \mathrm{~mol}^{-1}(\mathrm{PDI}=1.11)$ and $M_{\mathrm{n}, \mathrm{NMR}}=10200 \mathrm{~g} \mathrm{~mol}^{-1}$ for POEGA- $b$-PAA, $M_{\mathrm{n}, \mathrm{GPC}}=11000 \mathrm{~g}$ $\mathrm{mol}^{-1}(\mathrm{PDI}=1.16)$ and $M_{\mathrm{n}, \mathrm{NMR}}=10800 \mathrm{~g} \mathrm{~mol}^{-1}$ for POEGA- $b$ PMAEP, and $M_{\mathrm{n}, \mathrm{GPC}}=14100 \mathrm{~g} \mathrm{~mol}^{-1}(\mathrm{PDI}=1.17)$ and $M_{\mathrm{n}, \mathrm{NMR}}$ $=10800 \mathrm{~g} \mathrm{~mol}^{-1}$ for POEGA- $b$-PAMPS.

\section{Grafting of di-block copolymers onto the surface of upconversion nanoparticles}

UCNPs (20 mg) were dispersed in tetrahydrofuran (THF) (1 mL). A solution of each di-block copolymer $(50 \mathrm{mg})$ in THF $(2 \mathrm{~mL})$ was individually added in UCNP dispersion. The resulting dispersion was sonicated for one min, followed by incubation in a shaker overnight at $40{ }^{\circ} \mathrm{C}$. The polymer coated UCNPs were purified three times by centrifugation at $14000 \mathrm{rpm}$ for $10 \mathrm{~min}$. The supernatant was removed and the nanoparticles were redispersed in appropriate media such as water, MES ( $\mathrm{pH} 4.5$ ) and PBS ( $\mathrm{pH}$ 7.4) buffer for further investigation of their colloidal stability. The functionalized UCNPs were characterized using TGA, ATR-IR, TEM and DLS.

\section{Analytical instruments}

NMR spectroscopy. Monomer conversion and polymer compositions were characterized by ${ }^{1} \mathrm{H}$ NMR using Bruker Avance $300(300 \mathrm{MHz})$ spectrometers in $\mathrm{d}_{6}$-DMSO as solvents. All chemical shifts are quoted in parts per million (ppm), referenced to residual solvent frequencies ${ }^{1} \mathrm{H}$ NMR: $\mathrm{d}_{6}-\mathrm{DMSO}=$ 2.46 ppm. ${ }^{31} \mathrm{P}$ NMR spectra of the polymer was carried out in $\mathrm{d}_{6}$ DMSO for POEGA- $b$-PMAEP.

Monomer OEGA conversion $\left(\alpha^{\mathrm{OEGA}}\right)$ for the first block polymer was calculated using the following equation:

$$
\alpha^{\mathrm{OEGA}}=\left[\int_{4.1 \mathrm{ppm}} /\left(\int_{4.1 \mathrm{ppm}}+\int_{4.3 \mathrm{ppm}}\right)\right],
$$

with $\int_{4.1 \mathrm{ppm}}$ and $\int_{4.3 \mathrm{ppm}}$ corresponding to integral of the signal at 4.1 (polymer) and at 4.3 (monomer) ppm, respectively. The experimental molecular weight of the resultant polymer or block co-polymer can be calculated using the following equation:

$$
M_{\mathrm{n}, \mathrm{NMR}}^{\mathrm{POEGA}}=\alpha^{\mathrm{OEGA}} \times([\mathrm{OEGA}] /[\mathrm{RAFT}]) \times M_{\mathrm{w}}^{\mathrm{OEGA}}+M_{\mathrm{w}}^{\mathrm{RAFT}},
$$

where $M_{\mathrm{w}}^{\mathrm{OEGA}}$ and $M_{\mathrm{w}}^{\mathrm{RAFT}}$ are the molecular weight of monomer and RAFT agent, respectively.

Monomer conversions for the second blocks (AA, MAEP and AMPS) were also calculated from ${ }^{1} \mathrm{H}$ NMR spectra of the reaction mixture before and after polymerization by comparing the integral ratio of the vinyl protons of monomer and the unchanged methylene protons of the POEGA adjacent to ester bond at $4.1 \mathrm{ppm}$.

For example, AA conversion $\left(\alpha^{\mathrm{AA}}\right)$ was calculated using the following equation:

$$
\alpha^{\mathrm{AA}}=\left(\int_{6.2}-\int_{6.2 \mathrm{ppm}, 0(\mathrm{~h})}\right) / \int_{6.2 \mathrm{ppm}, 0(\mathrm{~h})}
$$

with the integrals at $4.1 \mathrm{ppm}$ were set at the same value before and after polymerization.

The experimental molecular weight of the resultant polymer or block co-polymer can be calculated using the following equation:

$$
\begin{aligned}
M_{\mathrm{n}, \mathrm{NMR}}^{\mathrm{POEGA}-\mathrm{PAA}}=\alpha^{\mathrm{AA}} & \times([\mathrm{AA}] /[\mathrm{POEGA} \text { macro-RAFT }]) \\
& \times M_{\mathrm{w}}^{\mathrm{AA}}+M_{\mathrm{n}, \mathrm{NMR}}^{\mathrm{POEGA}}
\end{aligned}
$$

where $M_{\mathrm{w}}^{\mathrm{AA}}$ and $M_{\mathrm{n}, \mathrm{NMR}}^{\mathrm{POEG}}$ are the molecular weight of monomer and macro RAFT agent, respectively.

The experimental molecular weights of the resultant POEGA$b$-PMAEP and POEGA- $b$-PAMPS block co-polymers were calculated using the similar above approach.

Size exclusion chromatography (SEC). Size exclusion chromatography (SEC) was implemented using a Shimadzu modular system comprising a DGU-12A degasser, a LC-10AT pump, a SIL10AD automatic injector, a CTO-10A column oven, a RID-10A refractive index detector, and a SPD-10A Shimadzu UV/vis detector. A $50 \times 7.8 \mathrm{~mm}$ guard column and four $300 \times$ $7.8 \mathrm{~mm}$ linear columns $(500,103,104$, and $105 \AA$ pore size, $5 \mu \mathrm{m}$ particle size) were used for the analyses. $N, N^{\prime}$-Dimethylacetamide (DMAc, HPLC grade, $0.05 \% \quad \mathrm{w} / \mathrm{v}$ 2,6-dibutyl-4methylphenol (BHT), $0.03 \% \mathrm{w} / \mathrm{v} \mathrm{LiBr}$ ) with a flow rate of 1 $\mathrm{mL} \min ^{-1}$ and a constant temperature of $50{ }^{\circ} \mathrm{C}$ was used as the mobile phase with an injection volume of $50 \mu \mathrm{L}$. The samples were filtered through $0.45 \mu \mathrm{m}$ filters. The unit was calibrated using commercially available linear polystyrene standards (0.5$1000 \mathrm{kDa}$, Polymer Laboratories). Chromatograms were processed using Cirrus 2.0 software (Polymer Laboratories).

Infrared spectroscopy. ATR-FTIR spectra of the polymer grafted upconversion nanoparticles were obtained using a Nicolet 7650 system using diffuse reflectance sampling accessories. The spectrophotometer was equipped by tungsten halogen lamp and Si/Ca beam splitter. Spectra were obtained at regular time intervals in the MIR region of $4000-500 \mathrm{~cm}^{-1}$ at a resolution of $4 \mathrm{~cm}^{-1}$ (128 scans) and analysed using OPUS software.

Dynamic light scattering (DLS). Dynamic light scattering studies of the upconversion nanoparticles at $0.5 \mathrm{mg} \mathrm{mL}^{-1}$ were conducted using a Malvern Instruments Zetasizer Nano ZS instrument equipped with a $4 \mathrm{mV}$ He-Ne laser operating at $\lambda=$ 
$633 \mathrm{~nm}$, an avalanche photodiode detector with high quantum efficiency, and an ALV/LSE-5003 multiple tau digital correlator electronics system.

Transmission electron microscopy (TEM). The size and dispersion of the upconversion nanoparticles was characterized using a FEI Tecnai G2 20 TEM with a beam voltage of $200 \mathrm{kV}$. Samples were prepared by placing a droplet of a $0.2 \mathrm{mg} \mathrm{mL}$ nanoparticles solution in water on carbon-coated copper grid.

X-ray powder diffraction (XRD). X-ray diffraction pattern measurements were conducted on a Bruker D8 Discover X-ray diffractometer equipped with $\mathrm{Cu} \mathrm{K} \alpha$ radiation from $5^{\circ}$ to $75^{\circ}$.

Thermal gravimetric analysis (TGA). TGA was performed on a SQ600. The samples were thoroughly purified by centrifuge to remove completely the unreacted polymers. Pre-dried samples were heated from room temperature to $700{ }^{\circ} \mathrm{C}$ at a constant temperature increase of $10{ }^{\circ} \mathrm{C} \min ^{-1}$ using air as the furnace gas. The weight loss was calculated through the difference between the weights at room temperature and at $600{ }^{\circ} \mathrm{C}$. The calculation of grafting density of polymers onto upconversion nanoparticles (in molecules per $\mathrm{nm}^{2}$ ) was described in ESI. $\dagger$

\section{Results and discussion}

The as-prepared upconversion nanoparticles are capped with hydrophobic oleic acid for both controlling the growth of nanocrystals during the synthesis and preventing the aggregation of the nanoparticles due to steric stabilization. For many practical applications, surface modification of nanoparticles is required for the dispersion of nanoparticles in aqueous solution. The grafting of polymer onto upconversion nanoparticles has been widely described in the literature ${ }^{15}$ It is very important to point out that the choice of the right anchoring molecules is critical for the stability of polymer coated UCNPs. Theoretically, the strongly binding anchoring molecules stabilize UCNPs better than weakly binding ones. The aim of this study is to experimentally compare the adsorption competition between polymers bearing phosphate, carboxylic acid and sulphonic acid with oleate ligand to provide a better understanding of the influence of functional anchoring groups on the stability of UCNPs.

\section{Synthesis of POEGA- $b$-PMAEP, POEGA- $b$-PAA, POEGA- $b$ - PAMPS di-block copolymer}

We employed RAFT polymerization techniques for the synthesis of three amphiphilic di-block copolymers $\left(\mathrm{POEGA}_{20}-b\right.$-PMAEP $_{5}$, POEGA $_{20}-b-\mathrm{PAA}_{5}$ and POEGA $20-b$-PAMPS ${ }_{5}$ ). These copolymers are composed of the same length of hydrophilic block polymer POEGA to render the biocompatibility and hydrophilicity, and a short hydrophobic block containing the five repeating units of phosphate, carboxylic acid and sulphonic acid functional groups (PMAEP, PAA and PAMPS) (Scheme 1). Poly(oligoethylene glycol) methyl ether acrylate (POEGA) macro-RAFT agent was prepared in toluene at $70{ }^{\circ} \mathrm{C}$ in the presence of BTPA RAFT agent and oligo(ethylene glycol) acrylate (OEGA) as a monomer. We determined around $90 \%$ of monomer conversion using ${ }^{1} \mathrm{H}$ NMR spectroscopy. It is necessary to stop the polymerization before the full conversion to avoid the formation of significant dead polymers that will create broader molecular weight distribution. Accordingly, the molecular weight of the POEGA macro-RAFT agent was measured to be $9800 \mathrm{~g} \mathrm{~mol}^{-1}$ with narrow distribution (PDI $=1.10$ ) by GPC and $M_{\mathrm{n}, \mathrm{NMR}}=9500 \mathrm{~g} \mathrm{~mol}^{-1}$ by ${ }^{1} \mathrm{H} \mathrm{NMR}$ ( $\sim 20$ repeating units) (ESI, Fig. S1†). POEGA macro-RAFT agent has been further used for subsequent polymerization with MAEP, AA and AMPS monomers to yield POEGA- $b$-PMAEP, POEGA- $b$-PAA, and POEGA- $b$-PAMPS di-block copolymer, respectively. The synthesis of block copolymers was stopped at the conversions of these monomers to be around $70 \%$ to yield the final di-block copolymers containing additional 5 repeating units of ligand functional groups. After purification, GPC and ${ }^{1} \mathrm{H}$ NMR spectra analysis confirmed the successful chains extension to a higher molecular weight with a good control of polydispersity (PDI < 1.20). We noted that the molecular weight determined by GPC for POEGA- $b$-PAA and POEGA- $b$-PAMPS are higher comparing with the values determined by ${ }^{1} \mathrm{H}$ NMR. This is due to the lack of retention of these acidic polymers onto the GPC columns and also the use of linear polystyrene calibration for data analysis. Both ${ }^{1} \mathrm{H}$ NMR spectra and ATR-FTIR have confirmed the presence of ester, phosphate, amide and sulphonic acid (ESI, Fig. S2-S6†). The FTIR spectra for the POEGA$b$-PAMPS polymer shows the characteristic signals of $\mathrm{S}=\mathrm{O}$ asymmetric and symmetric and $\mathrm{S}-\mathrm{O}$ stretches of sulphonic groups at 1355, 1150 and $750 \mathrm{~cm}^{-1}$ (ESI, Fig. S6†).

\section{Conjugation of POEGA- $b$-PMAEP, POEGA- $b$-PAA and POEGA- $b$-PAMPS di-block copolymers onto upconversion nanoparticles}

Oleate-capped $\mathrm{NaYF}_{4}: \mathrm{Yb} / \mathrm{Er}$ nanoparticles (UCNP@OA) were first synthesized following a previously reported protocol. ${ }^{\mathbf{1 4 , 3 0}}$ TEM images of UCNP@OA showed the formation of monodisperse nanoparticles with average size of $32 \mathrm{~nm}$ (Fig. 1).

The X-Ray Diffraction (XRD) pattern of the UCNPs revealed the formation of mainly the hexagonal phase structure as bulk $\beta-\mathrm{NaYF}_{4}$ (ESI, Fig. S7 $\dagger$ ). It can be seen from the TEM image that the as-prepared OA capped UCNPs are well-dispersed in cyclohexane with no sign of aggregation and narrow size distribution was proven by DLS analysis. The prepared upconversion nanoparticles were then functionalized with di-block copolymers through ligand exchange process. The presence of copolymers on the surface of upconversion nanoparticles was confirmed using ATR-FITR and TGA techniques. ATR-FTIR is a powerful technique to prove the attachment of polymers onto the surface of UCNPs. FTIR spectra of bare UCNP, UCNP@OA, and UCNP@polymers were shown in Fig. 2. All the absorptions of UCNP@OA were clearly identified, the $\mathrm{C}-\mathrm{H}$ stretching vibration bands of unsaturated $-\mathrm{C}=\mathrm{C}-\mathrm{H},-\mathrm{CH}_{3}$ and $-\mathrm{CH}_{2}$ - are located at 3000,2927 and $2852 \mathrm{~cm}^{-1}$ respectively, the $\mathrm{C}-\mathrm{H}$ bending vibration band appears at $1443 \mathrm{~cm}^{-1}$ and the asymmetric and symmetric COO- stretching at 1547 and $1435 \mathrm{~cm}^{-1}$ of oleic acid molecules.

Interestingly, after modifying with POEGA- $b$-PMAEP polymer bearing phosphate groups, the carboxylate peak of oleic acid at $1547 \mathrm{~cm}^{-1}$ completely disappeared, which is confirmed by the 


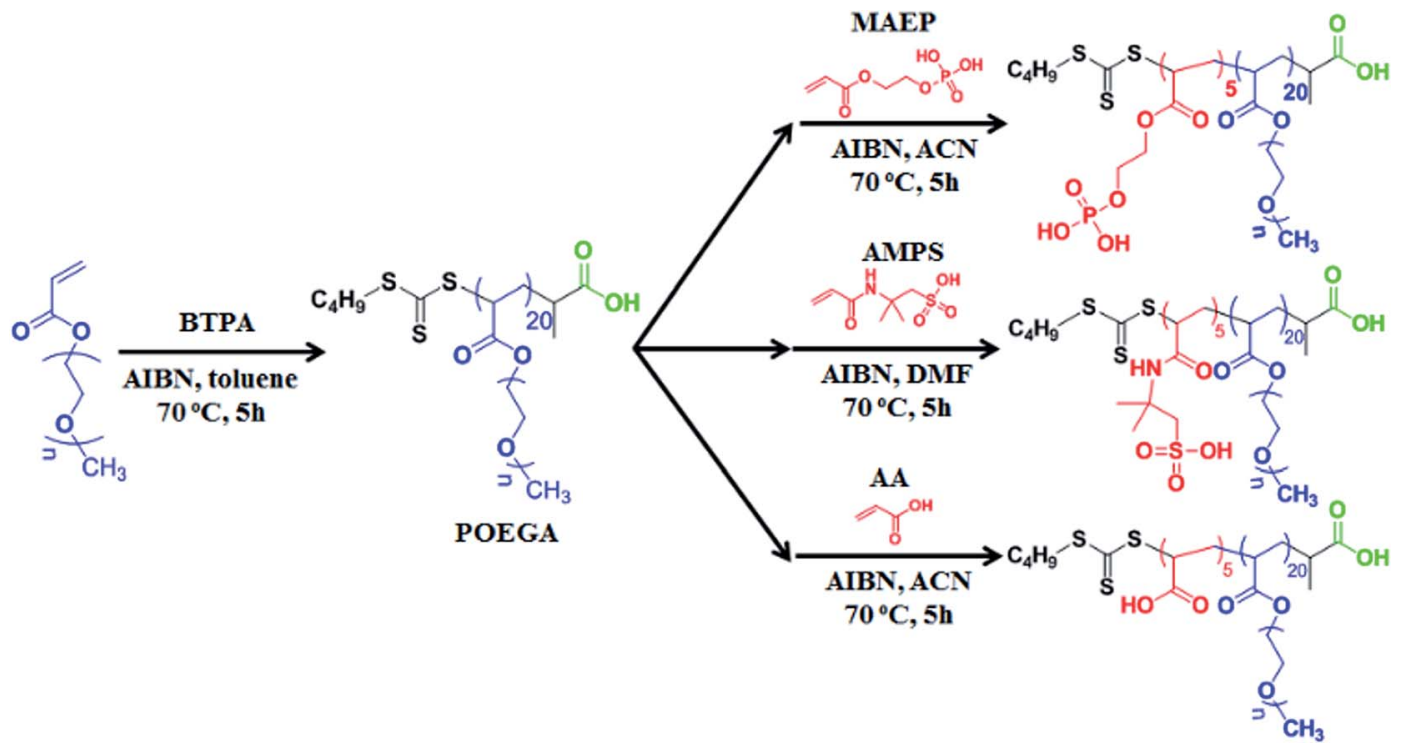

Scheme 1 POEGA- $b$-PMAEP, POEGA-b-PAMPS, and POEGA- $b$-PAA di-block copolymers via RAFT polymerization.
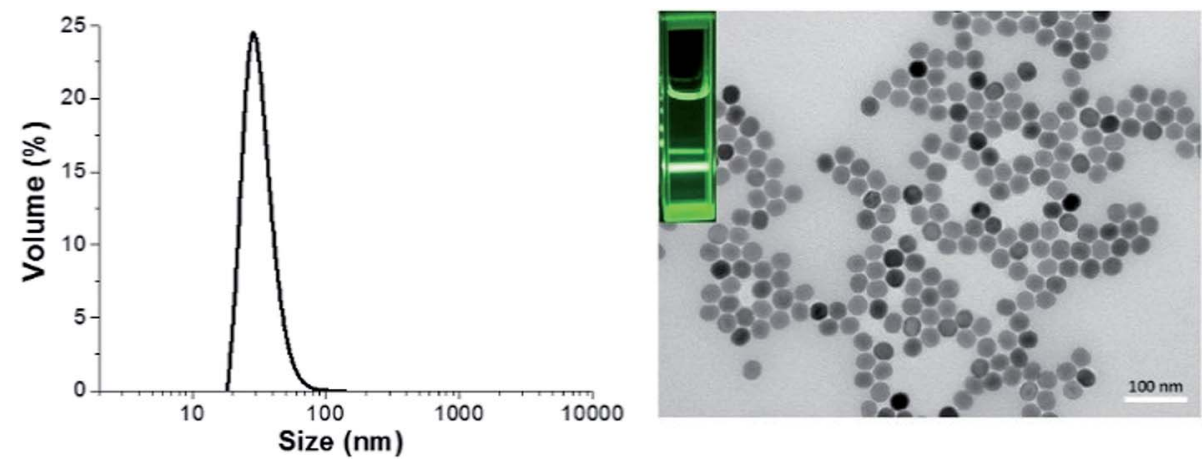

Fig. 1 Dynamic light scattering (DLS) graph (left) and TEM image (right) depicting the size of upconversion nanoparticles in cyclohexane, luminescence imaging (inset) under $980 \mathrm{~nm}$ excitation.

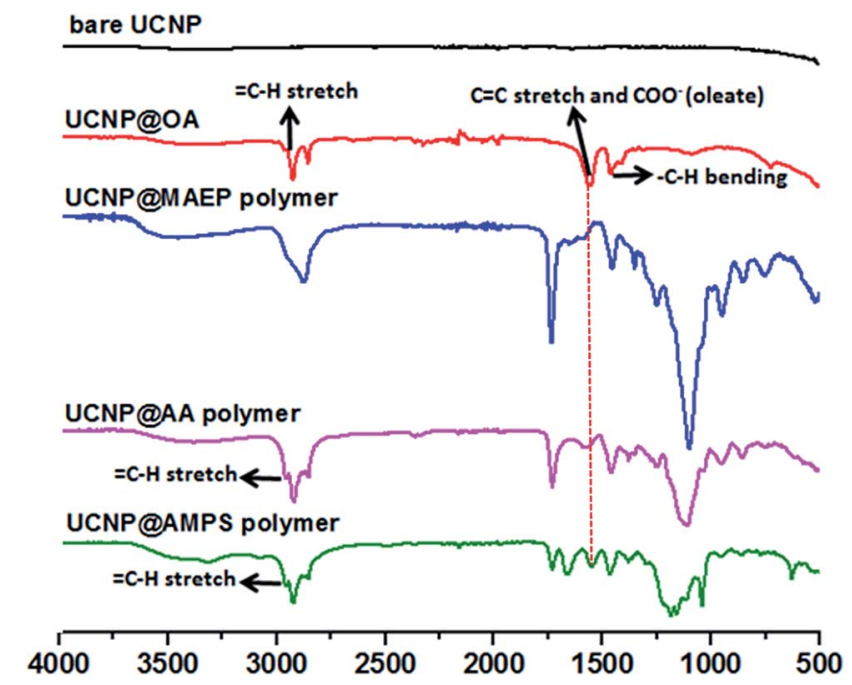

Fig. 2 ATR-FTIR spectra of prepared bare (OA removed) UCNP, UCNP@OA and UCNP@POEGA-b-PMAEP polymer, UCNPAPOEGA$b$-PAMPS polymer and UCNP@POEGA-b-PAA polymer. disappearance of alkene and carboxylate signals at 3000 and $1547 \mathrm{~cm}^{-1}$. In contrast, di-block polymers with carboxylic acid (POEGA- $b$-PAA) and sulphonic acid (POEGA- $b$-PAMPS) functional groups only could partially replace $\mathrm{OA}$ as evidenced by the presence of characteristic absorptions of remaining oleate as shown in Fig. 2. This result suggests that phosphate groups on the polymer have a higher affinity to the surface of nanoparticles. It is important to emphasize that the incomplete ligand exchange greatly influences the colloidal stability in aqueous media of final polymer coated UCNPs owing to the presence of hydrophobic oleic acid molecules. Regardless, the FTIR spectra confirms the successful grafting of all three functional polymers on the surface of UCNPs by the appearance of the characteristic absorptions, such as the characteristic $\mathrm{P}=\mathrm{O}$ stretches of POEGA- $b$-PMAEP $\left(1120 \mathrm{~cm}^{-1}\right), \mathrm{C}=\mathrm{O}$ stretches of POEGA- $b$-PAA $\left(1730 \mathrm{~cm}^{-1}\right)$ and $\mathrm{S}=\mathrm{O}$ and $\mathrm{S}-\mathrm{O}$ stretches of POEGA-b-PAMPS $\left(1355,1150\right.$ and $\left.750 \mathrm{~cm}^{-1}\right)$.

To quantitatively confirm the substitution amount of polymers on the surface of UCNPs, we performed thermogravimetric analysis (TGA) (ESI, Fig. S8 $\dagger$ ). The weight contribution 
of UCNP@OA, and UCNP@polymer nanoparticles is shown in Table 1. As the complete ligand exchange of oleic acid with POEGA- $b$-PMAEP polymer has been achieved, we are able to calculate the density of the adsorbed polymer (in molecules per $\mathrm{nm}^{2}$ ). Assuming the UCNP is spherical with the diameter of $32 \mathrm{~nm}$ and bulk density of $\mathrm{NaYF}_{4}$ is $4.2 \mathrm{~g} \mathrm{~cm}^{-3}$, the grafting density was calculated to be 6.73 for the OA molecules per $\mathrm{nm}^{2}$ for UCNP@OA and 0.29 polymer chains per $\mathrm{nm}^{2}$ for

Table 1 TGA results for UCNP@OA and UCNP@POEGA- $b$-PMAEP, UCNP@POEGA- $b-P A A$, and UCNP@POEGA- $b$-PAMPS

\begin{tabular}{lll}
\hline & $\begin{array}{l}\text { Weight loss } \\
(\mathrm{wt} \%)\end{array}$ & $\begin{array}{l}\text { Grafting density } \\
\left(\text { molecules per } \mathrm{nm}^{2}\right)\end{array}$ \\
\hline UCNP@OA & 12.35 & 6.73 \\
UCNP@POEGA- $b$-PMAEP & 14.16 & 0.29 \\
UCNP@POEGA- $b$-PAA & 15.32 & - \\
UCNP@POEGA- $b$-PAMPS & 17.81 & -
\end{tabular}

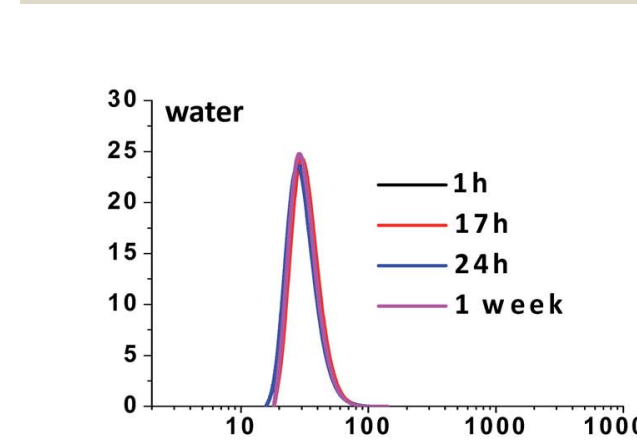

UCNP@POEGA- $b$-PMAEP. This is a predictable result as OA molecules 30 times lower in molecular weight than polymer and also more molecules can be adsorbed on the surface of the nanoparticles. The slightly higher weight loss for UCNP@POEGA- $b$-PAMPS and UCNP@POEGA- $b$-PAA could be attributed to the contribution of remaining oleic acid due to the incomplete ligand exchange.

\section{Colloidal stability of hybrid upconversion nanoparticles in different media}

In many applications of UCNPs, one of the most important prerequisites is a good colloidal stability of nanoparticles. Indeed, the UCNP@polymers hybrid nanostructures have shown a well-dispersed behavior in ethanol, THF and other organic solvents. In what follows, we assessed the colloidal stability of the polymer coated UCNPs not only in water, but also in physiologically relevant buffers, such as MES buffer ( $\mathrm{pH} 4.5)$ and PBS (pH 7.4) over one-week storage by measuring their

\section{UCNP@POEGA-b-PMAEP}
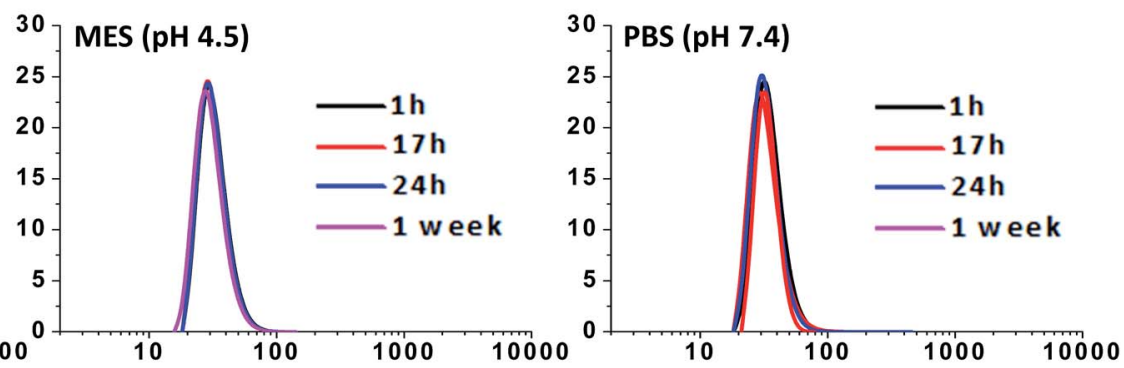

\section{UCNP@POEGA-b-PAA}
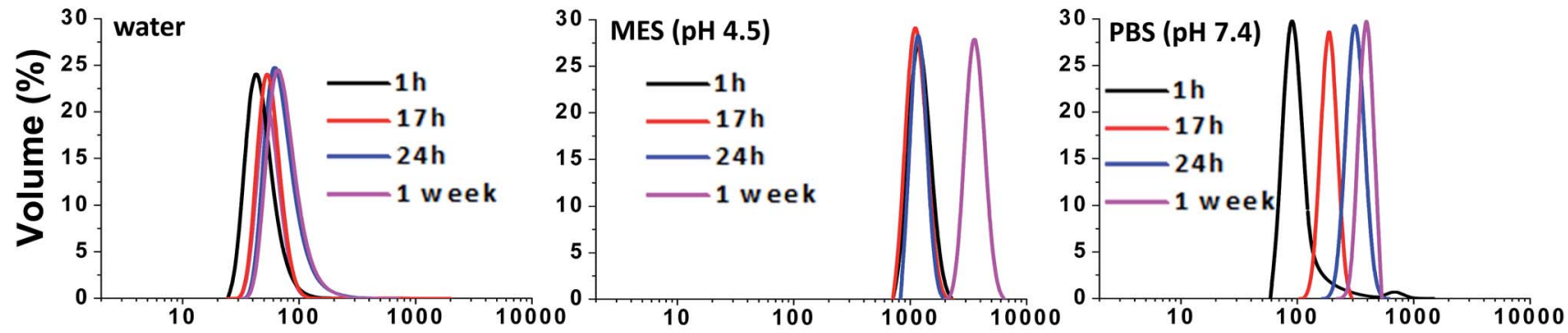

\section{UCNP@POEGA-b-PAMPS}
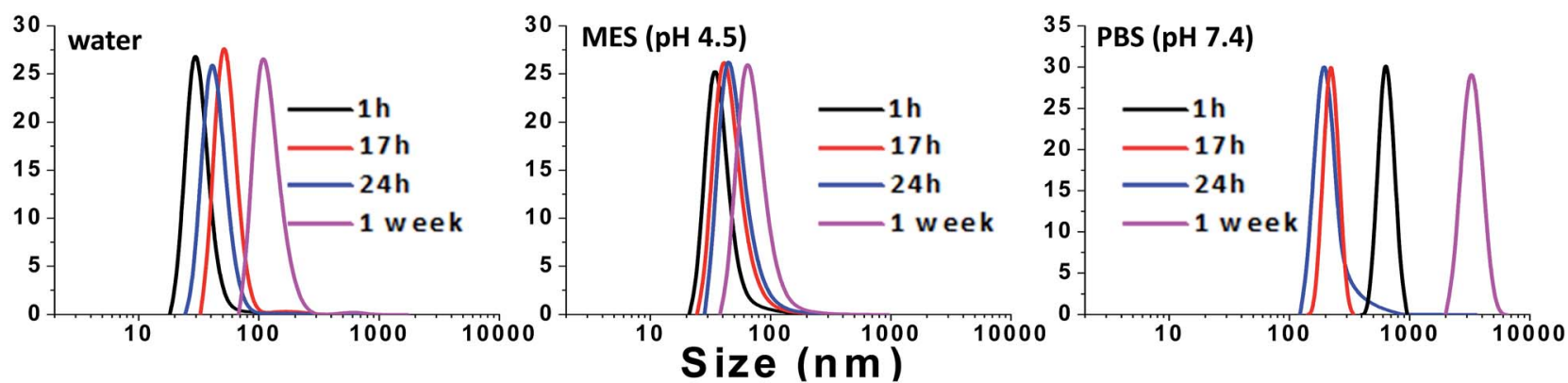

Fig. 3 Colloidal stability of UCNP@POEGA- $b$-PMAEP polymer, UCNP@POEGA- $b$-PAMPS polymer and UCNP@POEGA- $b-P A A$ polymer over one-week storage as determined by DLS measurements. 
hydrodynamic size using DLS. It is noted that the UCNPs can be easily dispersed in water immediately after the ligands exchange process with all three polymers and the solution is clear. After ligand exchange with POEGA- $b$-PMAEP, the UCNPs remained stable for one week without any sign of aggregation in water, PBS buffer and MES buffer (Fig. 3). The DLS plot showed the monodisperse distribution with the average size of about $38 \mathrm{~nm}$ after one week. Unexpectedly, the UNCPs capped with POEGA- $b$-PAA and POEGA- $b$-PAMPS, are only stable for the first few hours after transferring to water (Fig. 3). The amount of aggregation has increased over time with the shift to a higher hydrodynamic radius. After one day of storage, the UCNPs capped with POEGA- $b$-PAA and POEGA- $b$-PAMPS polymers tends to form aggregates which are visible to the naked eye. In this case, the intensity-based distribution is not fully meaningful as it only represents the large aggregates. Thus, volumebased distribution is used for the DLS data analysis to avoid the misinterpretation due to the presence of significant amount of aggregation. Both UNCPs capped with POEGA- $b$-PAA and POEGA- $b$-PAMPS have become very unstable with the severe formation of aggregation immediately after being transferred to PBS buffer ( $\mathrm{pH}$ 7.4). UNCPs capped with POEGA- $b$-PAMPS showed better stability in MES buffer ( $\mathrm{pH} 4.5)$ than ones capped with POEGA- $b$-PAA which could be explained by the higher acidity of sulphonic acid ( $\left.\mathrm{p} K_{\mathrm{a}} c a .-3\right)$ compared to carboxylic acid ( $K_{\mathrm{a}} c a$. 4.2) These DLS data demonstrated that UCNP@POEGA- $b$-PMAEP shows the best colloidal stability in aqueous media in comparison with UCNP@ POEGA- $b$-PAA and UCNP@ POEGA- $b$-PAMPS. The results correlate well with the FTIR data

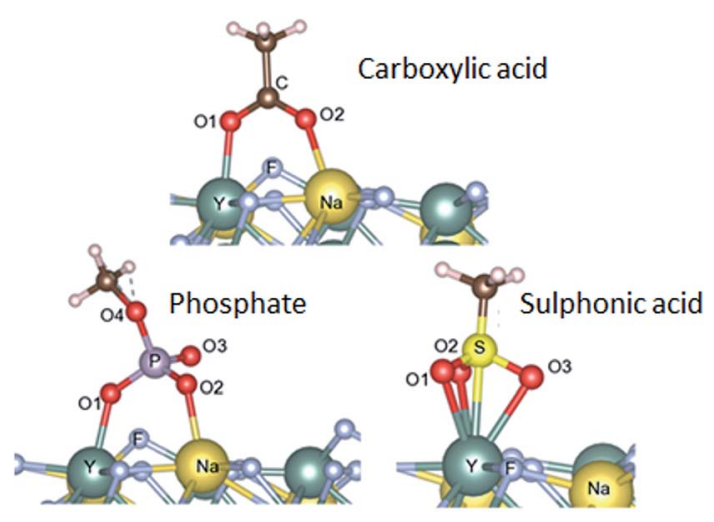

Fig. 4 Adsorption configurations for the three structures considered in our calculations. that show that UCNP@PAA and UCNP@PAMPS have a residual $\mathrm{OA}$ on the surface. The incomplete ligand exchange could be one of the reasons for the instability of UCNP@ POEGA- $b$-PAA and UCNP@ POEGA- $b$-PAMPS in aqueous media due to the hydrophobicity of OA fatty acid. Additionally, introduction of charged salt into aqueous solution also contributes to a charge screening between nanoparticles surface leading to further aggregation.

\section{Adsorption energy of ligand groups on UCNP surface}

It is very interesting that the chemical structures of the anchoring groups strongly influence the binding of polymers onto the UCNPs and therefore their colloidal stability. To further investigate the adsorption mechanism of different functional ligands onto the surface of UCNPs, we performed density functional theory (DFT) ${ }^{31}$ by calculating the adsorption energies between phosphate, carboxylic, sulphonic acid groups and lanthanide ions. Hartree Fock Density Functional Theory (HF-DFT) has been used to study the adsorption of phosphonic acid on $\mathrm{TiO}_{2}$ surface. ${ }^{32}$ DFT approach is usually much simpler than HF-DFT. Specifically, we simulated the interaction between the three anchoring groups and the (001) surface of $\beta$ $\mathrm{NaYF}_{4}$. The specific structure for the (001) plane (ESI, Fig. S9†) was selected based on its relatively low surface energy. ${ }^{\mathbf{1 4}}$ Details of our calculations for finding the relaxed structures are provided in the ESI. $\uparrow$ The optimal adsorption configurations of the various groups to the surface are shown in Fig. 4. Adsorption energies and bond lengths of the deprotonated groups to the (001) surface were calculated (Table 2). Carboxylic acid and phosphate groups are bonded to the surface via the bonding of two $\mathrm{O}$ atoms to the adjacent surface $\mathrm{Y}$ and $\mathrm{Na}$ atoms, with adsorption energies of $-77.9 \mathrm{kcal} \mathrm{mol}^{-1}$ and $-90.4 \mathrm{kcal} \mathrm{mol}^{-1}$ respectively. The sulphonic acid group bonds by having the three $\mathrm{O}$ atoms bonded to one surface $\mathrm{Y}$ atom, with the adsorption energy of $-80.0 \mathrm{kcal} \mathrm{mol}^{-1}$. It is noted that the $\mathrm{O}-\mathrm{Y}$ bond lengths (Table 2) for carboxylic acid and phosphate groups are generally lower that the corresponding $\mathrm{O}-\mathrm{Na}$ bond lengths, owing to the stronger bonding with the metal atoms. The adsorption of phosphate was calculated to be stronger by $12.5 \mathrm{kcal} \mathrm{mol}^{-1}$ than that of carboxylic acid group and by $10.4 \mathrm{kcal} \mathrm{mol}^{-1}$ than the sulphonic acid group. It is clear that the trend in adsorption energies agrees well with the experimental observation, where the phosphate anchor group is more competitive in binding to the surface of upconversion nanoparticles with the greatest colloidal stability.

Table 2 The adsorption energies of the three structures considered in the calculations and the bond lengths involving the $\mathrm{O}$ atoms and the $\mathrm{Y}$, $\mathrm{Na}$, $\mathrm{C}, \mathrm{P}$ and/or $\mathrm{S}$ atoms. Note that the three $\mathrm{O}$ atoms of the $\mathrm{SA}$-group are bonded to the $\mathrm{Y}$ atom, and therefore we list the corresponding bond lengths in the column with heading O1-Y. The S-Yt bond length (2.68 Å) is not included in this table

\begin{tabular}{|c|c|c|c|c|c|c|c|c|c|c|c|c|}
\hline Ligand group & $\begin{array}{l}\text { Adsorption energy } \\
\left(\mathrm{kcal} \mathrm{mol}^{-1}\right)\end{array}$ & \multicolumn{11}{|l|}{ Bond lengths $(\AA)$} \\
\hline Carboxylic acid & -77.9 & 2.23 & 2.29 & 1.3 & - & - & 1.26 & - & - & - & - & - \\
\hline Sulphonic acid & -80.0 & $2.51,2.50,2.48$ & - & - & - & 1.38 & - & - & 1.38 & - & 1.37 & - \\
\hline
\end{tabular}




\section{Conclusions}

The present work provides a fundamental understanding on the molecular mechanism of the competition between phosphate, carboxylic and sulphonic acid which are three widely used anchoring groups for surface modification of upconversion nanoparticles. The versatility of the RAFT polymerization technique allows us the precisely controlled composition of polymers with different ligands groups gaining an important advantage in this study and essential for conclusive comparison. Our result showed that the structural and chemical characteristics of ligand groups strongly impact the ligand exchange efficiency with OA capped UCNPs, which in turn influence the colloidal stability of the polymer capped UCNPs in aqueous media. Importantly, our experimental finding shows that phosphate ligands lead to the excellent stability of UCNPs for an extended period of time due the complete replacement of OA molecules on the surface and greater absorption energy for phosphate groups with lanthanide ions. These finding is important for many biological applications of UCNPs, especially for diagnostic and theranostics. This strategy of surface modification and functionalization would be also useful for other inorganic colloidal nanoparticles.

\section{Conflicts of interest}

There are no conflicts to declare.

\section{Acknowledgements}

The authors acknowledge the financial support from the Australian Research Council (ARC) Future Fellowship Scheme (D. J., FT 130100517) and ARC Industry Transformational Research Hub Scheme (IH150100028). O. S. acknowledges NHMRC-ARC Dementia Research Fellowship (APP1101258). Prof Cyrille Boyer, Nik Nik Mohd Adnan and Sivaprakash Shanmugam are kindly thanked for the help with GPC measurements.

\section{Notes and references}

1 F. Wang and X. Liu, Chem. Soc. Rev., 2009, 38, 976-989.

2 D. Jin, Nat. Photonics, 2016, 2016, 567-569.

3 X. Liu, C.-H. Yan and J. A. Capobianco, Chem. Soc. Rev., 2015, 44, 1299-1301.

4 Z. Hou, K. Deng, C. Li, X. Deng, H. Lian, Z. Cheng, D. Jin and J. Lin, Biomaterials, 2016, 101, 32-46.

5 D. Yang, C. Li and J. Lin, Nanomedicine, 2015, 10, 2573-2591. 6 D. J. Gargas, E. M. Chan, A. D. Ostrowski, S. Aloni, M. V. P. Altoe, E. S. Barnard, B. Sanii, J. J. Urban, D. J. Milliron, B. E. Cohen and P. J. Schuck, Nat. Nanotechnol., 2014, 9, 300-305.

7 Y. Liu, Y. Lu, X. Yang, X. Zheng, S. Wen, F. Wang, X. Vidal, J. Zhao, D. Liu, Z. Zhou, C. Ma, J. Zhou, J. A. Piper, P. Xi and D. Jin, Nature, 2017, 543, 229-233.

8 A. Bagheri, H. Arandiyan, C. Boyer and M. Lim, Adv. Sci., 2016, 3, 1500437.
9 X. Ai, C. J. H. Ho, J. Aw, A. B. E. Attia, J. Mu, Y. Wang, X. Wang, Y. Wang, X. Liu, H. Chen, M. Gao, X. Chen, E. K. L. Yeow, G. Liu, M. Olivo and B. Xing, Nat. Commun., 2016, 7, 10432.

10 H. Dong, S.-R. Du, X.-Y. Zheng, G.-M. Lyu, L.-D. Sun, L.-D. Li, P.-Z. Zhang, C. Zhang and C.-H. Yan, Chem. Rev., 2015, 115, 10725-10815.

11 N. Goswami, Q. Yao, Z. Luo, J. Li, T. Chen and J. Xie, J. Phys. Chem. Lett., 2016, 7, 962-975.

12 X.-R. Song, N. Goswami, H.-H. Yang and J. Xie, Analyst, 2016, 141, 3126-3140.

13 H. T. Whelan, E. V. Buchmann, A. Dhokalia, M. P. Kane, N. T. Whelan, M. T. T. Wong-Riley, J. T. Eells, L. J. Gould, R. Hammamieh, R. Das and M. Jett, J. Clin. Laser Med. Surg., 2003, 21, 67-74.

14 D. Liu, X. Xu, Y. Du, X. Qin, Y. Zhang, C. Ma, S. Wen, W. Ren, E. M. Goldys, J. A. Piper, S. Dou, X. Liu and D. Jin, Nat. Commun., 2016, 7, 10254.

15 V. Muhr, S. Wilhelm, T. Hirsch and O. S. Wolfbeis, Acc. Chem. Res., 2014, 47, 3481-3493.

16 G. Zhao, L. Tong, P. Cao, M. Nitz and M. A. Winnik, Langmuir, 2014, 30, 6980-6989.

17 A. Sedlmeier and H. H. Gorris, Chem. Soc. Rev., 2015, 44, 1526-1560.

18 Y. Que, C. Feng, G. Lu and X. Huang, ACS Appl. Mater. Interfaces, 2017, 9, 14647-14655.

19 I. Recalde, N. Estebanez, L. Frances-Soriano, M. Liras, M. Gonzalez-Bejar and J. Perez-Prieto, Nanoscale, 2016, 8, 7588-7594.

20 G. Moad, E. Rizzardo and S. H. Thang, Polymer, 2008, 49, 1079-1131.

21 H. T. Duong, V. T. Huynh, P. de Souza and M. H. Stenzel, Biomacromolecules, 2010, 11, 2290-2299.

22 T.-K. Nguyen, H. T. T. Duong, R. Selvanayagam, C. Boyer and N. Barraud, Sci. Rep., 2015, 5, 18385.

23 H. T. T. Duong, C. P. Marquis, M. Whittaker, T. P. Davis and C. Boyer, Macromolecules, 2011, 44, 8008-8019.

24 K. Knop, R. Hoogenboom, D. Fischer and U. S. Schubert, Angew. Chem., Int. Ed., 2010, 49, 6288-6308.

25 L. Tao, C. S. Kaddis, R. R. Ogorzalek Loo, G. N. Grover, J. A. Loo and H. D. Maynard, Chem. Commun., 2009, 21482150.

26 W. Scarano, H. T. T. Duong, H. Lu, P. L. De Souza and M. H. Stenzel, Biomacromolecules, 2013, 14, 962-975.

27 C. Boyer, V. Bulmus, T. P. Davis, V. Ladmiral, J. Liu and S. Perrier, Chem. Rev., 2009, 109, 5402-5436.

28 T.-K. Nguyen, R. Selvanayagam, K. K. K. Ho, R. Chen, S. K. Kutty, S. A. Rice, N. Kumar, N. Barraud, H. T. T. Duong and C. Boyer, Chem. Sci., 2016, 7, 1016-1027.

29 C. J. Ferguson, R. J. Hughes, D. Nguyen, B. T. T. Pham, R. G. Gilbert, A. K. Serelis, C. H. Such and B. S. Hawkett, Macromolecules, 2005, 38, 2191-2204.

30 H.-S. Qian and Y. Zhang, Langmuir, 2008, 24, 12123-12125.

31 J. P. Perdew, K. Burke and M. Ernzerhof, Phys. Rev. Lett., 1996, 77, 3865-3868.

32 M. Nilsing, S. Lunell, P. Persson and L. Ojamäe, Surf. Sci., 2005, 582, 49-60. 